\title{
Multiplexed Single Photons from Deterministically Positioned Nanowire Quantum Dots
}

\author{
Zhe-Xian Koong $\odot,{ }^{1, *}$ Guillem Ballesteros-Garcia, ${ }^{1}$ Raphaël Proux $\odot,{ }^{1}$ Dan Dalacu, ${ }^{2}$ Philip J. Poole $\odot,{ }^{2}$ \\ and Brian D. Gerardot ${ }^{1, \dagger}$ \\ ${ }^{1}$ SUPA, Institute of Photonics and Quantum Sciences, Heriot-Watt University, Edinburgh EH14 4AS, \\ United Kingdom \\ ${ }^{2}$ National Research Council of Canada, Ottawa, Ontario K1A 0R6, Canada
}

(Received 11 May 2020; revised 13 July 2020; accepted 7 August 2020; published 3 September 2020)

\begin{abstract}
Solid-state quantum emitters are excellent sources of on-demand indistinguishable or entangled photons and can host long-lived spin memories, crucial resources for photonic quantum-information applications. However, their scalability remains an outstanding challenge. Here, we present a scalable technique to multiplex streams of photons from multiple independent quantum dots, on chip, into a fiber network for use "off chip." Multiplexing is achieved by incorporating a multicore fiber into a confocal microscope and spatially matching the multiple foci, seven in this case, to quantum dots in an array of deterministically positioned nanowires. As a proof-of-principle demonstration, we perform parallel spectroscopy on the nanowire array to identify two nearly identical quantum dots at different positions, which are subsequently tuned into resonance with an external magnetic field. Multiplexing of background-free single photons from these two quantum dots is then achieved. Our approach, applicable to all types of quantum emitters, can readily be integrated into scalable photonic based quantum technologies.
\end{abstract}

DOI: 10.1103/PhysRevApplied.14.034011

\section{INTRODUCTION}

Motivated by the phenomenal scalability of semiconductor integrated circuits for classical computing and communication, semiconductor quantum photonic chips have been pursued for light-based quantum-information technologies. Visions of fully integrated quantum photonic chips, which require on-demand indistinguishable single-photon generation integrated on chip with low-loss directional couplers, phase shifters, filters, and singlephoton detectors, have been presented [1-4]. On-chip integration of solid-state emitters such as color centers in diamond [5-8], molecules $[9,10]$, two-dimensional materials [11-14], and III-V semiconductor quantum dots (QDs) [15-21], are particularly promising for these applications. As solid-state emitters reach maturity, the next logical step is to interface these sources with larger quantum photonic architectures to promote the scalability and realization of multipartite quantum-information protocols [22]. For example, near-term application of

\footnotetext{
*zk49@hw.ac.uk

†b.d.gerardot@hw.ac.uk

Published by the American Physical Society under the terms of the Creative Commons Attribution 4.0 International license. Further distribution of this work must maintain attribution to the author(s) and the published article's title, journal citation, and DOI.
}

quantum-information protocols such as quantum key distribution [23] (including measurement-deviceindependent schemes [24]), boson sampling [25,26], and photonic cluster state generation for measurement-based quantum computing [27-29] all benefit from having multiple streams of indistinguishable photons that can be realized with QDs. However, while each independent ingredient of the fully integrated chip vision has been realized separately, hybrid integration of all components in a fully functional platform is a demanding long-term challenge. Hence, in the near term, a viable approach to develop applications is to connect the separate components via optical interlinks such as optical fibers. In addition, long-term applications such as long-distance quantum communication [30] or distributed quantum networking [31,32] necessitate coupling of the quantum light off chip into a fiber network.

To handle the capacity and connectivity required for these applications, multiplexing techniques that expand the current solid-state hardware infrastructure are required. One approach is the integration of multicore fibers (MCFs) in a fiber-based quantum optical network. MCFs have $n$ cores within a single cladding and achieve single-mode transmission of each core with minimal crosstalk [33]. These properties enable an ultrahigh (Tb/s) optical transmission capacity [34] and spatial-division multiplexing, with up to $n=120$ cores [35] for optical quantum communication [36]. 
In this paper, we introduce a multispot microscopy setup that combines two MCFs for individual excitation and collection of multiple foci in a confocal microscope. By matching the foci from each fiber core to a customdesigned chip of spatially positioned nanowire arrays with embedded QDs, we are able to probe multiple independent quantum emitters (up to $n=7$ ) on a single chip and collect the fluorescence from each quantum emitter into a separate single-mode fiber. By connecting the MCF to a linear fiber array at the input of an imaging spectrometer, we are able to simultaneously perform spectroscopy on the seven independent QDs in the array. We take advantage of this to rapidly scan the quantum-emitter array and identify two QDs with ground-state emissions separated by just 0.113 $\mathrm{meV}$, which we subsequently tune into resonance with an external magnetic field. By exciting both QDs individually, we observe suppressed multiphoton emission probability in the emission from both QDs, which validates the generation of single photons from remote emitters on the same chip. This constitutes a proof-of-principle demonstration of multiplexing single-photon streams from separate quantum sources. Our results motivate the integration of both coherent excitation and parallel spectroscopy to generate streams of indistinguishable single photons from multiple emitters on the same chip, which are crucial resources for photonic quantum technologies.

\section{LOW-TEMPERATURE MULTISPOT CONFOCAL-MICROSCOPE SETUP}

Figure 1(a) shows the schematic of the multispot microscope. We use two commercial MCFs (OptoScribe, COF073400, core diameter $8.2 \mu \mathrm{m}$ ) consisting of $n=7$ cores arranged in a hexagonal pattern with a lattice constant of $\left.a_{0}=35 \mu \mathrm{m}\right)$. The fan-outs connected to each of the MCFs, at the excitation and collection, allow us to excite and collect from each focus independently. Our microscope builds on a previous design using only a MCF in the collection arm of the microscope [37] that prevents independent excitation and collection at each focus. The MCFs are mounted on rotation cages to allow precise alignment of the excitation and collection spots. The lateral displacement of the beam from the MCF, collimated using a collimating lens (Thorlabs C280TMD-B, focal length $f_{1}=18.4 \mathrm{~mm}$ and $\left.\mathrm{NA}=0.15\right)$, is compensated with a pair of relay lenses (RL1 and RL2) to form a quasi- $4 f$ configuration. The relay lenses, both singlets, have focal lengths of $f_{2}=250 \mathrm{~mm}$ (RL1) and $f_{3}=300 \mathrm{~mm}$ (RL2). They are positioned $50 \mathrm{~cm}$ above the microscope objective (focal length $f_{\mathrm{obj}}=2.93 \mathrm{~mm}$ and NA $=0.81$ ). This gives an estimated magnification of $M=f_{\text {obj }} f_{3} / f_{1} f_{2} \approx 0.19$ and an estimated spot-size separation of $a=M a_{0} \approx 6.7 \mu \mathrm{m}$. Upon illumination of all seven cores of the MCF using the same 940-nm (1.319-eV) narrow-band laser, we observe an image of the MCF on the camera (CCD). The image,

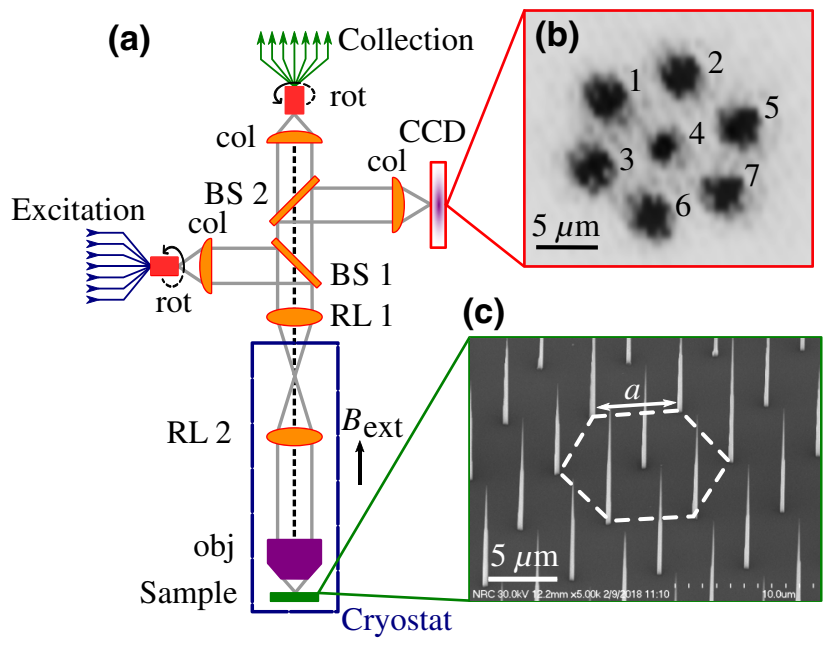

FIG. 1. The multispot microscope and nanowire sample structure. (a) A schematic of the lens configuration of the multispot microscope: col, collimating lens; rot, rotatable fiber mount; BS, 96:4 beam splitter; RL, relay lens; obj, microscope objective; CCD, charged-coupled device. (b) An image of the collection or excitation spots on the CCD device. (c) A SEM image of the tilted sample. The nanowires are arranged in a hexagonal lattice (the geometry is outlined by the dashed lines) with lattice spacing $a$.

shown in Fig. 1(b), gives a measured spot spacing of $6.5 \mu \mathrm{m}$, with the slight deviation from the estimated value due to a small inaccuracy in the position of the relay lenses.

In this work, we utilize InP nanowires deterministically positioned in a hexagonal lattice with the lattice constant (a) ranging from $6.1 \mu \mathrm{m}$ to $7 \mu \mathrm{m}$. Figure 1(c) shows a scanning electron microscope (SEM) image of the tilted nanowire sample. The range of lattice constants is designed to optimize the spatial overlap between the foci of the multicore fiber to the position of the nanowires when accounting for a small deviation in the magnification from the expected value. Within each nanowire, (In,As)P QDs with ground-state emission from 910 to $950 \mathrm{~nm}$ are embedded. Further details of the growth process are described in Refs. [38,39]. The sample is kept at $T=3.7 \mathrm{~K}$ in a closedcycle cryostat (Attocube attoDry1000) equipped with a 9-T superconducting magnet. The sample is mounted in the Faraday configuration, where the external magnetic field $\left(B_{\text {ext }}\right)$ is parallel to the nanowire growth axis. For antibunching experiments, a tunable band-pass filter with a full width at half maximum of $1 \mathrm{~nm}(1.47 \mathrm{meV})$, centered at $916.30 \mathrm{~nm}(1.3531 \mathrm{eV})$ is placed before the collimating lens at the collection to filter out the above-band continuous-wave (cw) excitation laser at $830 \mathrm{~nm}(1.4938$ $\mathrm{eV}$ ) and any unwanted emission lines. These filtered photons, coupled into the MCF in the collection, are directed to a Hanbury Brown-Twiss (HBT) type interferometer. The fan-out coupler with the collected photon streams is coupled to a 50:50 nonpolarizing fiber beam splitter. The fiber 
beam splitter outputs are then sent to a pair of superconducting nanowire single-photon detectors (SNSPD), each with a nominal timing jitter and detection efficiency of approximately 100 ps and approximately $90 \%$ at $950 \mathrm{~nm}$, respectively.

To analyze the signal from all seven outputs from the collection MCF simultaneously on a spectrometer, we mount the MCF fan-out to a custom-designed fiber array holder (FAH) in front of the entrance slit of an imaging spectrometer. The schematic of the fluorescence detection setup is illustrated in Fig. 2(a). The FAH ensures that all of the outputs from the microscope (labeled from 1 to 7) can be imaged on the CCD array $(1340 \times 100$ pixels $)$ simultaneously, with their corresponding region of interests on the CCD array (labeled as $a$ to $f$ ) vertically displaced relative to each other. We characterize the crosstalk matrix of the full system (the multispot microscope and the imaging spectrometer setup combined) by injecting laser light into the microscope through one of the excitation fibers and measuring the signal received at each of the detection spots on the spectrometer. The crosstalk matrices for all seven cores are summarized in Fig. 2(b). Despite showing near-dark-count performance (nominal crosstalk of approximately $-35 \mathrm{~dB}$ ) for most of the pixels on the $\mathrm{CCD}$, the crosstalk measured on adjacent pixels indicates a nonideal nominally about $-15 \mathrm{~dB}$ leakage between adjacent spots. The crosstalk between channels stems from imperfect focus at the sample, arising from the multimode behavior of each fiber core at $\lambda=940 \mathrm{~nm}$, which inevitably couples scattered laser light into neighboring spots, as well as due to the leakage between adjacent regions of interest on the spectrometer's CCD array. Here, we partially mitigate this by illuminating nonadjacent collection or excitation spots where the detection suffers from the least crosstalk. By illuminating three out of seven of the excitation spots, we can perform clean spectroscopy on three spatially separated nanowire QDs simultaneously, as shown in Fig. 2(c). This demonstrates the ability to collect fluorescence from multiple nanowire QDs concurrently using the multispot microscope and the spectroscopy setup successfully achieves spatial multiplexing from multiple emitters.

\section{SPECTROSCOPY OF NANOWIRE QUANTUM DOTS}

Our candidate of choice to demonstrate spatially multiplexed streams of single photons from solid-state emitters is a sample of (In,As)P QDs embedded in deterministically positioned InP nanowires [40]. Nanowire QDs offer an excellent coherent light-matter interface [41] and can emit high-quality entangled photons $[42,43]$, while providing near-unity coupling into optical fiber due to their wave-guiding properties $[44,45]$ and Gaussian-modeprofile emission [46]. This gives a nominal extraction efficiency of $18 \%$, as reported previously [42,43]. They are deterministically grown with up to five QDs on the same nanowire [47] and can be controllably positioned on integrated photonic chips $[48,49]$.

To characterize our sample, we first perform spectroscopy on a single-nanowire QD. For this characterization, we replace the multispot microscope setup with a confocal microscope. The scattered photons are then coupled into a single-mode fiber and sent to a spectrometer for spectral characterization, a single-photon detector for time-resolved lifetime measurement, or a HBT-type interferometer for second-order correlation measurement. We record the time tags from each detector and extract the coincidences at various detection time delays, $\tau$ [50].
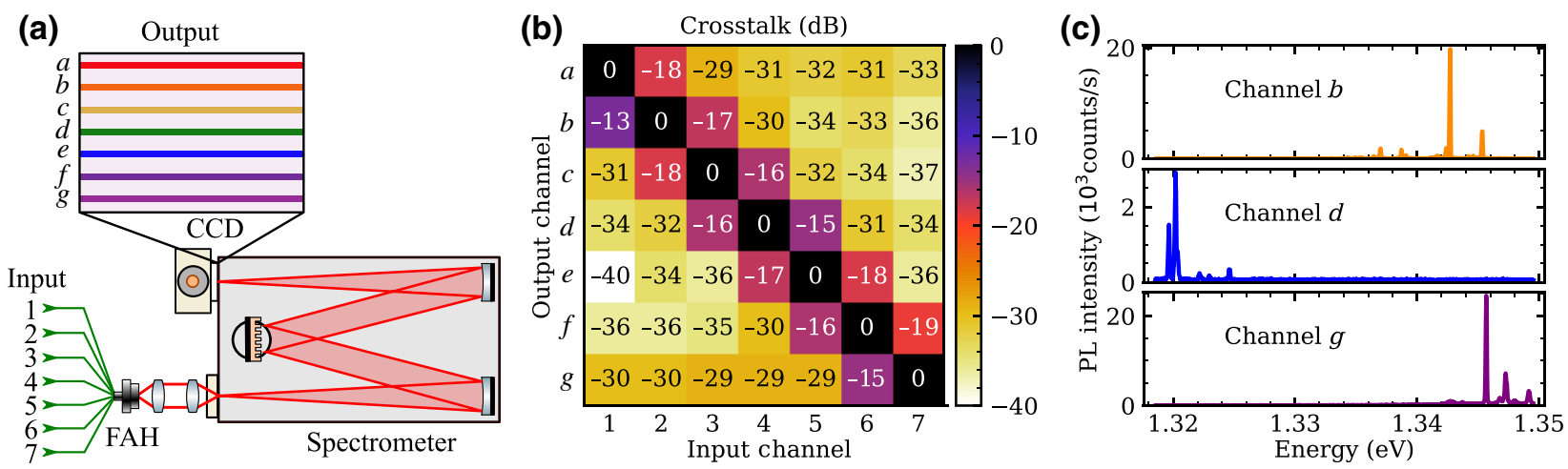

FIG. 2. The multiplexed spectroscopy. (a) A schematic of the setup for fluorescence detection on the spectrometer. The MCF fanouts (labeled from 1 to 7) are coupled into an imaging spectrometer via a fiber array holder (FAH). The multispot signals are imaged on a CCD detector array $(1340 \times 100$ pixels), with a separate region of interest, labeled from $a$ to $f$, indicated on the CCD. (b) The crosstalk matrix between the input fiber fan-out and the output pixels on the spectrometers. The adjacent channels show crosstalk of approximately $-15 \mathrm{~dB}$, while the rest of the channels show crosstalk of approximately $-35 \mathrm{~dB}$, approaching the dark counts of the spectrometer. (c) An example of the emission spectra from three nanowires (corresponding to fluorescence at channels $b, d$, and $g$ ) measured simultaneously. 
By focusing the cw above-band (1.4938-eV) excitation laser on a single nanowire, we obtain the photoluminescence (PL) emission spectrum from a single QD, shown in Fig. 3(a). The PL spectrum shows multiple emission lines, with the neutral exciton $X$, the biexciton $X X$, and the negatively charged exciton $X^{1-}$ each exhibiting spectrometerresolution-limited line widths $(\mathrm{FWHM}=38 \mu \mathrm{eV})$. We identify the nature of the emission lines via polarizationresolved measurements. This is done by replacing the quarter-wave plate with a half-wave plate on the collection path and then, by rotating the half-wave plate with a piezoelectric rotator, recording the PL spectra at each rotation angle, $\theta$. We fit the data with $\omega_{X, X X}=\omega_{0}+(\Delta / 2) \sin (\theta+$ $\phi)$, where $\omega_{0}$ is the mean emission energy, $\Delta / 2$ is the amplitude and $\phi$ is the phase of the oscillation. The amplitude extracted from the fit gives an exciton fine-structure splitting of $\Delta=11.2 \mu \mathrm{eV}$, while the difference in the mean emission energy of the $X$ and $X X$ gives the biexciton binding energy $E_{B}=1.861 \mathrm{meV}$. Here, we identify the $X X$ and $X$ pair via the out-of-phase oscillation in the emission energy as a function of the linear polarization angle $\theta$, which implies linear and orthogonally polarized emissions. The emission line corresponding to the $X^{1-}$ does not have a fine-structure splitting and hence is circularly polarized. These results are shown as the inset in Fig. 3(a).

Using a free-space spectral filter (spectral bandwidth of approximately $100 \mu \mathrm{eV}$ ), we selectively filter the emission from either the $X$ or the $X X$ transition. Time-resolved measurements on both transitions, when exciting with a pulsed source (approximately 3-ps pulse width, $80.3-\mathrm{MHz}$ repetition rate, and pulse area of $\pi$ ) via resonant twophoton excitation [51-53], give a radiative lifetime of $T_{1}^{X}=902(1) \mathrm{ps}$ and $T_{1}^{X X}=507(1)$ ps for the $X$ and $X X$ emissions, respectively. The results are shown in Fig. 3(b). The fitting details for the time-resolved lifetime measurement are included in Appendix A.
Figure 3(c) shows the power dependence in the detected count rate of the $X$ and $X X$ emissions under cw resonant two-photon excitation. At low excitation power, the intensity of the $X$ and $X X$ emissions increases with the excitation power, until they reach saturation at $3 \mu \mathrm{W}$, giving a saturation detected count rate of 1.1 and $1.3 \times 10^{6}$ counts/s on the SNSPD, respectively. Additional spectroscopy results obtained under resonant two-photon excitation are described in Appendix B, showing coherent population control and high-purity single-photon generation.

\section{MULTIPLEXED SINGLE-PHOTON SOURCES}

Having characterized our source, we now aim to illustrate the full functionality of our microscope by multiplexing the single-photon streams of two degenerate QDs. Here, the ability to probe multiple nanowires in parallel accelerates the search for pairs of QDs emitting at similar emission energy in the nanowire arrays. We target our search for a pair of QDs within the tuning range of approximately $0.3 \mathrm{meV}$ allowed via Zeeman shift for a maximum applied magnetic field of $B_{\text {ext }}=9 \mathrm{~T}$. We address the nanowires in the multispot microscope using aboveband (1.4938-eV) excitation and find two QDs with similar exciton emissions (centered at $1.3531 \mathrm{eV}$ ) with an energy difference of $0.113 \mathrm{meV}$. We estimate that the probability of finding a pair of QDs in this sample emitting at similar energy within a single scan of $n=3$ QDs is $\alpha_{3}=1 \%$. This value scales as $n(n-1) / 6 \times \alpha_{3}$ for $n>3$, which increases the success rate to $7 \%$ when operating at maximum capacity (i.e., spectroscopy with all $n=7$ cores). The emission spectra for both of the near-degenerate QDs, before (original) and after (filtered) spectral filtering, are shown in Figs. 4(a) and 4(b). These QDs are labeled by their excitation spot (spots 2 and 7), as indicated in the inset of Fig. 4(a). By applying an external magnetic field, $B_{\text {ext }}$, we (a)

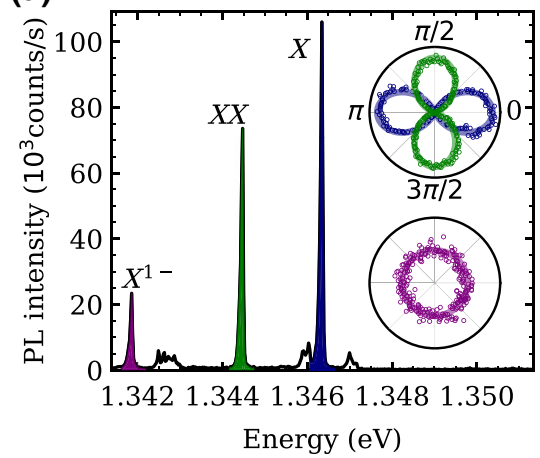

(b)

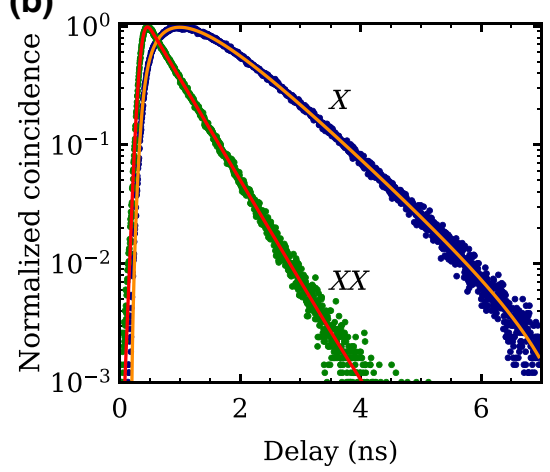

(c)

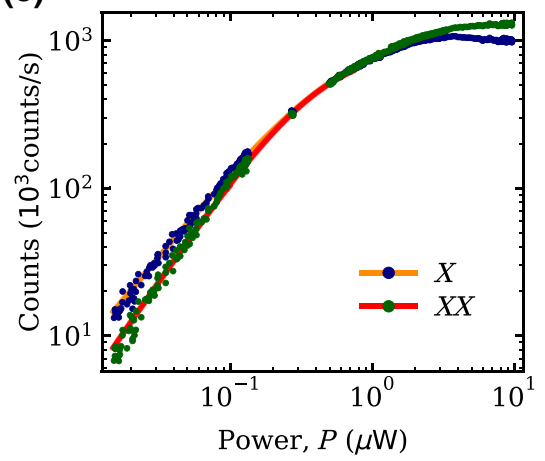

FIG. 3. The spectroscopy of a nanowire QD. (a) Emission spectra under above-band excitation (at $1.4938 \mathrm{eV}$ ), showing the emission lines from the $X, X X$, and $X^{1-}$ transitions. The insets show the emission energy as a function of the polarization angle, confirming the nature of each emission line ( $X$, blue; $X X$, green; $X^{1-}$, purple). (b) The lifetime measurement of the biexciton-exciton cascade under resonant two-photon excitation. (c) The power dependence of the $X$ and $X X$ emissions under cw resonant two-photon excitation. 

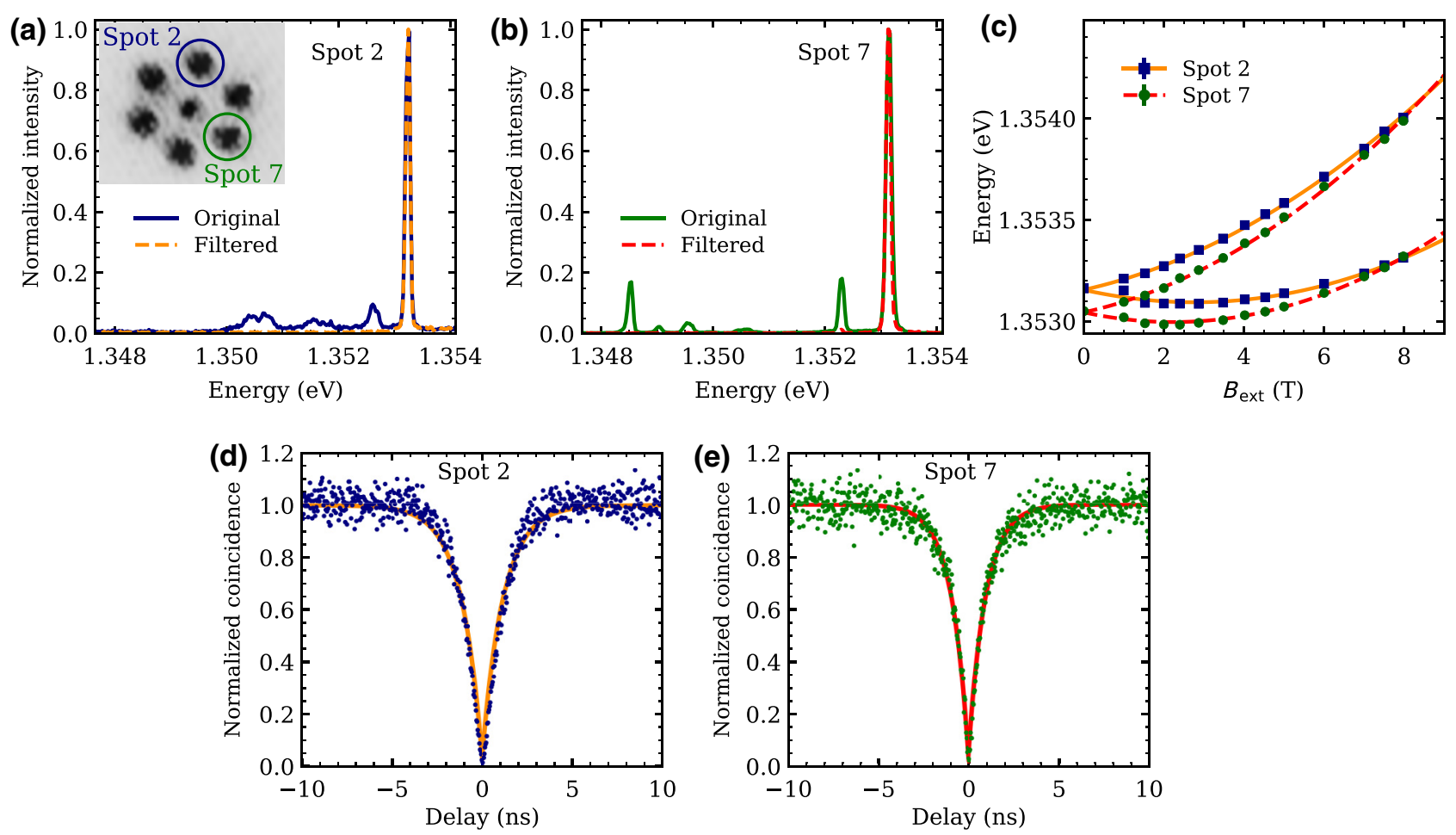

FIG. 4. Multiplexing single photons from independent quantum emitters. (a),(b) Emission spectra of the original (solid line) and filtered (dashed line) from wires at spot 2 (c) and spot 7 (d), under above-band excitation. The inset figure indicates the excitation and collection spots for wires at spots 2 and 7. (c) The emission energy of the filtered QDs as a function of the external magnetic field, $B_{\text {ext }}$. The fits give a $g$ factor and a diamagnetic shift coefficient of 1.53(1) and 7.99(7) $\mu \mathrm{eV} / \mathrm{T}^{2}$ for the QDs at spot 2 (solid line) and 1.49 (1) and 9.65(8) $\mu \mathrm{eV} / \mathrm{T}^{2}$ at spot 7 (dashed line). (d),(e) The normalized $g^{(2)}$ for the emission from nanowires at spot 2 (f) and spot $7(\mathrm{~g})$, at $B_{\text {ext }}=0$, showing a suppressed multiphoton emission of $g^{(2)}(0) \sim 0$.

can fine tune and minimize the difference in the emission energies of both QDs. This result is shown in Fig. 4(c), where their emission energies match at $B_{\text {ext }} \approx 8 \mathrm{~T}$. The fits yield a $g$ factor of $1.53(1.49)$ and a diamagnetic shift coefficient of 7.99(9.65) $\mu \mathrm{eV} / \mathrm{T}^{2}$ for the QD at spot 2 (spot 7). The ability to tune two emitters on the same chip into resonance (the same emission energy) can enable two-photon interference experiments from remote emitters on the same chip, effectively reducing the resource overhead by eliminating the need for additional cryogenic setups for this purpose.

To verify high-purity multiplexed single-photon emission from these QDs, we perform HBT measurements on the emissions from the two QDs. Their corresponding coincidence histograms, $g^{(2)}$, are shown in Figs. 4(d) and 4(e). Fitting the $g^{(2)}$ data with Eq. (1)

$$
g^{(2)}(\tau)=1-\xi \exp \left(-|\tau| / T_{1}\right)
$$

gives a vanishing multiphoton emission probability of $g^{(2)}(0)=1-\xi \sim 0$ for both QDs at spots 2 and 7. The decay times, $T_{1}$, extracted from the $g^{(2)}$ histograms are similar for both QDs: $T_{1}=1.14(1) \mathrm{ns}$ for the QD at spot 2 and $T_{1}=0.92(1) \mathrm{ns}$ for the QD at spot 7 . The detected count rates for these measurements (exciting at below saturation) are 84 and $60 \times 10^{3}$ counts/s for the QDs at spots 2 and 7, respectively. These results certify the functionality of this microscope, as we demonstrate high-purity single-photon multiplexing from emitters on the same chip.

\section{DISCUSSION AND OUTLOOK}

A few straightforward improvements could be made to our multispot microscope setup. One issue to address is the strong chromatic aberrations introduced by the collimating and relay lenses, which cause the light from the above-band excitation laser to be defocused at the plane of the nanowires (optimized for the collection wavelength) and lead to crosstalk between excitation spots. To improve this, a cryostat that allows a large field of view without relay lenses (e.g., the Montana Cryostation or Attocube attoDry800) or achromatic lenses could be used. There is a second issue: the multimode behavior of the commercial MCFs at the QD emission wavelengths (910-950 nm) leads to extra crosstalk, poor excitation laser extinction in the dark-field configuration, and losses when coupling the 
MCF into single-mode fibers for interferometry or detection. Custom-fabricated MCFs that are single mode at the QD wavelength would alleviate these problems. Finally, an increase in the number of cores in the MCF is desirable to maximize scalability.

Nevertheless, we successfully demonstrate multiplexing of single photons from degenerate nanowire QDs using the multispot microscope. As a proof of principle, we manage to tune two emitters into resonance, which opens up the possibility of two-photon interference measurement from remote emitters on the same chip. The parallel spectroscopy enabled by our multispot microscope setup accelerates the characterization of these sources for future applications.

Spectral fluctuations, likely originating from charge noise from the solid-state environment and at the surface of the nanowire, degrade the performance of our specific photon source and prevent high-visibility twophoton interference experiments [54]. Hence, multiplexing of indistinguishable single or entangled photon streams would benefit from optimized quantum-emitter devices, which would ideally include deterministic positioning, near-perfect extraction efficiency, in situ tunability of each emitter's emission energy, minimal spectral fluctuations, and Purcell-enhanced emitter lifetimes. Additionally, easy incorporation of coherent optical control is necessary. Our choice of nanowire QDs, motivated here by the deterministic positioning and high extraction efficiency, can potentially be individually tuned using strain [55-57] but the dangling bonds at the nanowire sidewalls likely will remain an obstacle to reduce charge noise and the nanowire geometry hinders resonant laser excitation in the dark-field configuration. Ideally, the emitters would be incorporated into a heterostructure device with individual contacts for each spatial position in the array to provide charge control, control of the emitter energy, charge-environment stabilization, and suppression of solid-state environmental noise [58,59]. While micropillar- and bullseye-type devices based on self-assembled QDs [15-17] offer high extraction efficiency and strong Purcell enhancement, deterministic positioning remains a significant challenge to be overcome. On the other hand, waveguide architectures that can couple light into and out of remotely located quantum emitters could be ideal. Such a platform offers high extraction efficiency, significant Purcell enhancement, charge control, the ability for local strain tuning, and the freedom to guide light anywhere on chip to and from randomly positioned QDs [20,21]. However, the generation of high-visibility two-photon interference from remote QDs is a significant challenge to be met in all approaches [6062]. Regardless of the chosen nanophotonics platform, our multispot microscope enables genuine scalability by providing individual excitation and collection to multiplex streams of single photons for optical quantum-information processes.

\section{ACKNOWLEDGMENTS}

This work was supported by the EPSRC (Grants No. EP/L015110/1, No. EP/M013472/1, and No. EP/ P029892/1), the ERC (Grant No. 725920), and the EU Horizon 2020 research and innovation program under Grant Agreement No. 820423. B.D.G. thanks the Royal Society for a Wolfson Merit Award and the Royal Academy of Engineering for a Chair in Emerging Technology.

\section{APPENDIX A: LIFETIME MEASUREMENTS}

We proceed to fit the coincidence histogram from the time-resolved lifetime measurements with the following function:

$\mathcal{I}_{1}\left(\tau, \Gamma_{1}, \sigma\right)=\exp \left(\frac{\Gamma_{1}\left(\Gamma_{1} \sigma^{2}-2 \tau\right)}{2}\right) \operatorname{erfc}\left(\frac{\Gamma_{1} \sigma^{2}-\tau}{\sqrt{2} \sigma}\right)$,

$$
\mathcal{I}_{2}\left(\tau, \Gamma_{1}, \Gamma_{2}, \sigma\right)=A \mathcal{I}_{1}\left(\tau, \Gamma_{1}, \sigma\right)+B \mathcal{I}_{1}\left(\tau, \Gamma_{2}, \sigma\right) .
$$

Here, $\mathcal{I}_{1}\left(\tau, \Gamma_{1}, \sigma\right)$ is simply a Heaviside step function, defined such that at positive time, the function is consists of a single exponential function, with decay time $\tau_{1}=1 / \Gamma_{1}$, convoluted with the instrument response function (modeled as a Gaussian with standard deviation $\sigma$ ). The fitting parameters $A$ and $B$ in $\mathcal{I}_{2}\left(\tau, \Gamma_{1}, \Gamma 2, \sigma\right)$ represent the weighted contribution of the two exponential decays.

For exciton $X$ emission, the data are fitted with Eq. (A2), which consists of two exponential decays, with time scales $T_{X}$ and $T_{X X} . T_{X}$ and $T_{X X}$ correspond to radiative lifetime of the excition and biexciton, respectively. For biexction $X X$ emission, the data are fitted with the same equation, which consists of a single time scale $T_{X X}$.

\section{APPENDIX B: COHERENT CONTROL OF NANOWIRE QUANTUM DOTS VIA RESONANT TWO-PHOTON EXCITATION}

We perform spectroscopy on a single-nanowire QD, focusing on the emission from the biexciton-exciton cascade. The energy-level schematic of the four-level cascade system is described in Fig. 5(a). Upon excitation into the biexciton state, a cascaded radiative decay from biexciton $|X X\rangle$ to vacuum ground state $|0\rangle$ is triggered via either of the two exciton states $\left|X_{H, V}\right\rangle$. This generates a pair of polarization-entangled photons, orthogonally polarized in the horizontal $(H)$ and vertical $(V)$ linear polarized basis, provided that the fine-structure splitting, $\Delta$, is kept smaller than the exciton lifetime [63].

Here, we demonstrate resonant two-photon excitation (TPE) on the same nanowire QD. We employ a linear 
(a)

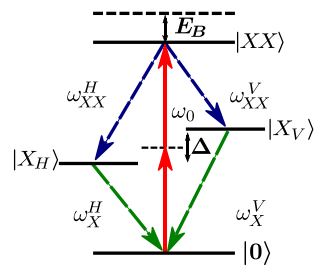

(c)

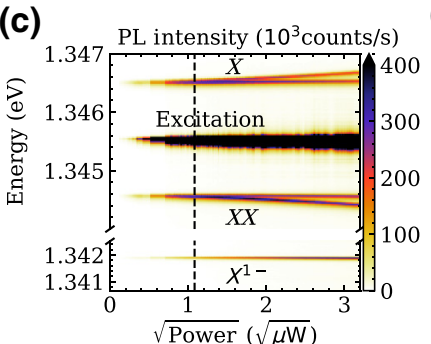

(e)
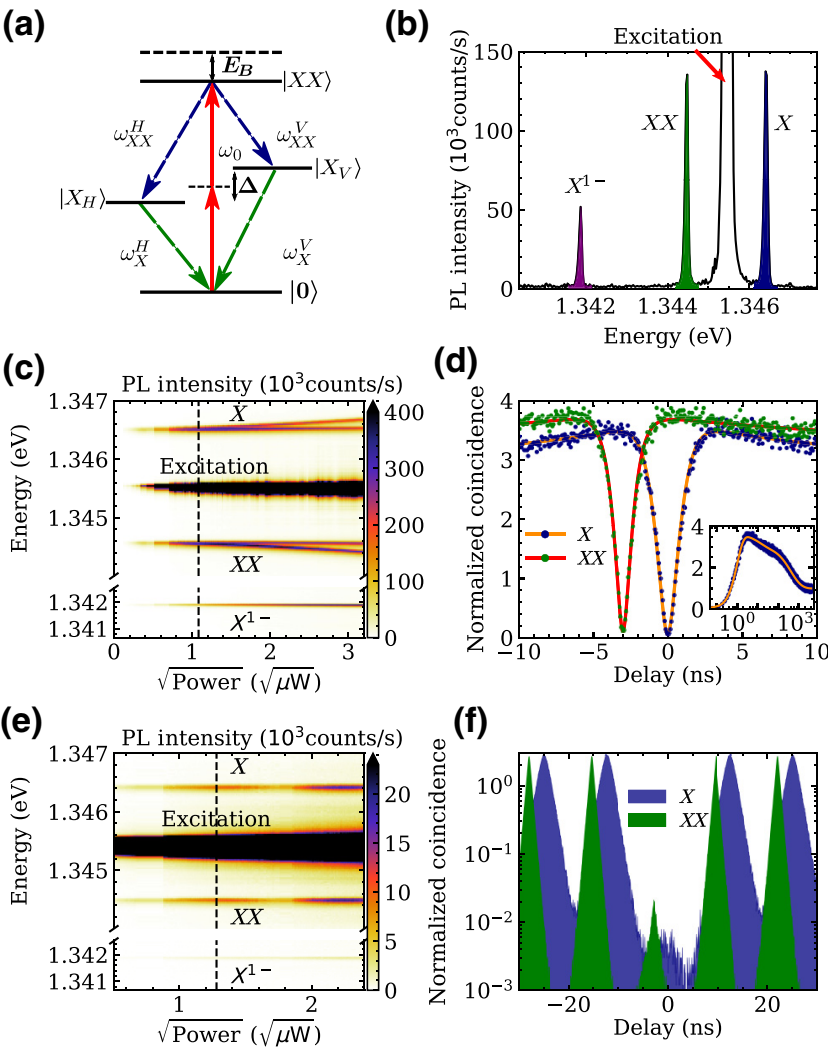

(d)

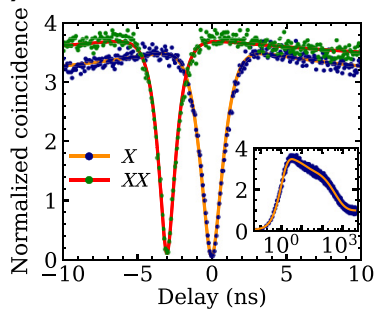

(f)

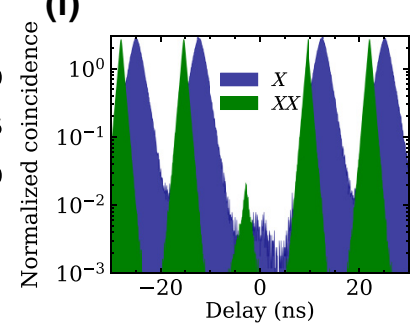

FIG. 5. The spectroscopy of a nanowire QD under resonant two-photon excitation (TPE). (a) An energy-level schematic of the biexciton-exciton $(X X-X)$ cascaded system: $\Delta$, exciton finestructure splitting; $E_{B}$, biexciton binding energy; $\omega_{0}$, excitation energy under resonant two-photon excitation; $\omega_{X, X X}$, emission energy for exciton $X$ and biexciton $X X$. (b) Emission spectra under resonant TPE (at $\omega_{0}=1.3455 \mathrm{eV}$ ) reveals cleaner emission lines of $X, X X$, and $X^{1-}$. (c) The power dependence of the emission under cw TPE reveals an Autler-Townes splitting for both $X$ and $X X$ emissions. (d) The second-order intensity correlation histogram, $g^{(2)}$, of the $X$ and $X X$ emissions [at a power $1.17 \mu \mathrm{W}$ indicated by the dashed line in (c)]. The inset shows the same $g^{(2)}$ of the $X$ emission, up to $5 \mu$ s. (e) The power dependence of the $X$ and $X X$ emissions under pulsed TPE reveals Rabi oscillations, with the $\pi$ pulse given by the black dashed line at $1.6 \mu \mathrm{W}$. (f) The $g^{(2)}$ data for $X$ and $X X$ emissions under $\pi$-pulse excitation [with the power indicated by the dashed line in (e)] in $\log$ scale. The $g^{(2)}$ data, normalized by the raw coincidence at $5 \mu \mathrm{s}$, for $X X$ emission in (d) and (f), are artificially shifted by -3 ns for clarity.

polarizer at the excitation and a quarter-wave plate and linear polarizer at the collection [64]. The linear polarizers are cross polarized to suppress the excitation laser. The quarter-wave plate is used to correct for any birefringence in the optical setup, which improves the polarization suppression. To perform TPE, we resonantly pump the population from the ground state $|0\rangle$ to the biexciton state $|X X\rangle$ with an excitation energy set to exactly half of the energy difference between the two states. This gives the TPE excitation energy of $\omega_{0}=E_{X X-0} / 2=\omega_{X}-$ $E_{B} / 2=1.3455 \mathrm{eV}$. We then resolve one of the exciton fine structures $\left|X_{H, V}\right\rangle$ by adjusting the linear polarizer in the collection to the polarization axis of the desired transition. At the same time, the linear polarizer in the excitation is aligned orthogonal to the collection linear polarizer to suppress the excitation power. The PL spectrum of the same QD under TPE is shown in Fig. 5(b). The scattered excitation laser, indicated on the figure, is a result of imperfect polarization suppression of the laser in the dark-field microscope, mostly caused by the geometry of the tip of the nanowire. Due to the resonant nature of the excitation, only the emission from the relevant transitions $\left(X, X X\right.$, and $\left.X^{1-}\right)$ is observed. In contrast with the PL spectrum in Fig. 3(a), the emission spectrum under TPE is much cleaner, indicating minimal excitation into the higher-energy states.

Figure 5(c) shows the power dependence in the PL emission spectra under $\mathrm{cw}$ excitation. At low excitation power, the intensity of the $X$ and $X X$ emissions increases with excitation power, until they reach saturation at $3 \mu \mathrm{W}$. Beyond the saturation, further increase in the excitation power reveals Autler-Townes splitting in the emission of both the $X$ and $X X$ transitions $[65,66]$. This has been previously demonstrated in Refs. [67-69] as a consequence of optical dressing of biexciton $|X X\rangle$ and ground $|0\rangle$ states. The formation of dressed states confirms the coherent nature of the excitation scheme, allowing for coherent manipulation of the emission from the biexciton-exciton cascade.

By fixing the excitation power at the saturation power, indicated by the black dashed line in Fig. 5(c), we perform HBT measurement on the $X$ and $X X$ emissions. Their normalized coincidence histograms $g^{(2)}$ are shown in Fig. 5(d). The inset of Fig. 5(d) shows the long time scale, up to $5 \mu \mathrm{s}$, of the normalized $g^{(2)}$ coincidences. The raw coincidences are normalized to the value at the longtime limit at $5 \mu \mathrm{s}$, where it is unaffected by the bunching in the $g^{(2)}$. Accounting for the multiple bunching time scales present in the $g^{(2)}$ measurement, we fit the data with

$$
g_{X(X X)}^{(2)}(\tau)=g_{\text {Resonant }}^{(2)}(\tau) \prod_{j \in\{a, b, c\}}\left(1+j e^{-\tau / \tau_{j}}\right),
$$

where $a, b$, and $c$ are the bunching amplitudes for the corresponding bunching time scales $\tau_{a}, \tau_{b}$, and $\tau_{c}$. The theoretical function $g_{\text {Resonant }}^{(2)}(\tau)$ follows the expression for a resonantly driven two-level system [70]. The resulting $g^{(2)}$ fit function is the convolution of Eq. (B1) with a Gaussian instrument-response function (FWHM $=160 \mathrm{ps})$. The fit shows excellent agreement with the experimental data, giving a suppressed multiphoton emission probability of $g_{X}^{(2)}(0)=0.006(1)$ and $g_{X X}^{(2)}(0)=0.023(2)$. The fit also reveals different bunching amplitudes and time scales for both the $X$ and the $X X$ emissions. The bunching in $g^{(2)}$ is 
TABLE I. The bunching time scale and amplitude, extracted from the fit to the $g^{(2)}$ data for both exciton $X$ and biexciton $X X$ emissions. The error, given by the standard deviation extracted from the fit, is included in brackets.

\begin{tabular}{lcc}
\hline \hline & Exciton, $X$ & Biexciton, $X X$ \\
\hline$\tau_{a}(\mathrm{~ns})$ & $12(1)$ & $19(1)$ \\
$\tau_{b}(\mathrm{~ns})$ & $391(3)$ & $376(1)$ \\
$\tau_{c}(\mathrm{~ns})$ & $769(6)$ & $992(9)$ \\
$a$ & $0.193(4)$ & $0.100(2)$ \\
$b$ & $0.717(16)$ & $1.432(12)$ \\
$c$ & $0.789(17)$ & $0.408(8)$ \\
\hline \hline
\end{tabular}

related to the spectral fluctuations of the emitter, likely due to charge noise in the QD environment and at the nanowire surface. The fit values of the bunching amplitudes and time scales of the $g^{(2)}$ for both the $X$ and the $X X$ emissions are summarized in Table I.

Figure 5(e) shows the power dependence in the PL emission spectra under pulsed resonant two-photon excitation. By varying the average excitation power of the pulsed source, we observe clear Rabi oscillations in the intensity of both the $X$ and the $X X$ emissions. We fit the data with a damped sinusoidal function to extract the power that corresponds to a $\pi$ pulse at $P_{\pi}=1.6 \mu \mathrm{W}$. We attribute the increase in counts in the $3 \pi$ pulse to be a result of experimental imperfection, most likely originating from pulse chirping [71,72] or slight detuning from the ideal resonant two-photon excitation energy, $\omega_{0}[73,74]$.

Next, we perform a pulsed HBT measurement by exciting the QD using a $\pi$ pulse $\left(P_{\pi}=1.6 \mu \mathrm{W}\right)$. The result is shown in Fig. 5(f), where we obtain a similar multiphoton emission probability of $g^{(2)}(0)=0.0117(4)$ and $g^{(2)}(0)=$ 0.0291 (5) for the $X$ and the $X X$ emission, respectively. The raw coincidences are normalized to the peaks at $5 \mu \mathrm{s}$, giving a long-bunching time scale of approximately 300 ns. Nevertheless, the observation of the Rabi oscillation and a vanishing $g^{(2)}(0)$ demonstrate coherent control of the state population as well as highly pure single-photon emission from the nanowire QDs.

[1] P. Lodahl, S. Mahmoodian, and S. Stobbe, Interfacing single photons and single quantum dots with photonic nanostructures, Rev. Mod. Phys. 87, 347 (2015).

[2] C. P. Dietrich, A. Fiore, M. G. Thompson, M. Kamp, and S. Höfling, GaAs integrated quantum photonics: Towards compact and multi-functional quantum photonic integrated circuits: GaAs integrated quantum photonics, Laser Photon. Rev. 10, 870 (2016).

[3] S. Bogdanov, M. Y. Shalaginov, A. Boltasseva, and V. M. Shalaev, Material platforms for integrated quantum photonics, Opt. Mater. Express 7, 111 (2017).
[4] J.-H. Kim, S. Aghaeimeibodi, J. Carolan, D. Englund, and E. Waks, Hybrid integration methods for on-chip quantum photonics, Optica 7, 291 (2020).

[5] S. L. Mouradian, T. Schröder, C. B. Poitras, L. Li, J. Goldstein, E. H. Chen, M. Walsh, J. Cardenas, M. L. Markham, D. J. Twitchen, M. Lipson, and D. Englund, Scalable Integration of Long-Lived Quantum Memories into a Photonic Circuit, Phys. Rev. X 5, 031009 (2015).

[6] Y.-C. Chen, P. S. Salter, S. Knauer, L. Weng, A. C. Frangeskou, C. J. Stephen, S. N. Ishmael, P. R. Dolan, S. Johnson, B. L. Green, G. W. Morley, M. E. Newton, J. G. Rarity, M. J. Booth, and J. M. Smith, Laser writing of coherent colour centres in diamond, Nat. Photonics 11, 77 (2016).

[7] B. Machielse, S. Bogdanovic, S. Meesala, S. Gauthier, M. J. Burek, G. Joe, M. Chalupnik, Y. I. Sohn, J. Holzgrafe, R. E. Evans, C. Chia, H. Atikian, M. K. Bhaskar, D. D. Sukachev, L. Shao, S. Maity, M. D. Lukin, and M. Lončar, Quantum Interference of Electromechanically Stabilized Emitters in Nanophotonic Devices, Phys. Rev. X 9, 031022 (2019).

[8] N. H. Wan, T.-J. Lu, K. C. Chen, M. P. Walsh, M. E. Trusheim, L. De Santis, E. A. Bersin, I. B. Harris, S. L. Mouradian, I. R. Christen, E. S. Bielejec, and D. Englund, Large-scale integration of artificial atoms in hybrid photonic circuits, Nature 583, 226 (2020).

[9] S. Grandi, M. P. Nielsen, J. Cambiasso, S. Boissier, K. D. Major, C. Reardon, T. F. Krauss, R. F. Oulton, E. A. Hinds, and A. S. Clark, Hybrid plasmonic waveguide coupling of photons from a single molecule, APL Photonics 4, 086101 (2019).

[10] D. Rattenbacher, A. Shkarin, J. Renger, T. Utikal, S. Götzinger, and V. Sandoghdar, Coherent coupling of single molecules to on-chip ring resonators, New J. Phys. 21, 062002 (2019).

[11] A. Branny, S. Kumar, R. Proux, and B. D. Gerardot, Deterministic strain-induced arrays of quantum emitters in a two-dimensional semiconductor, Nat. Commun. 8, 15053 (2017).

[12] J. E. Fröch, S. Kim, N. Mendelson, M. Kianinia, M. Toth, and I. Aharonovich, Coupling HBN quantum emitters to 1D photonic crystal cavities, arXiv:1911.02722 (2019).

[13] F. Peyskens, C. Chakraborty, M. Muneeb, D. V. Thourhout, and D. Englund, Integration of single photon emitters in 2D layered materials with a silicon nitride photonic chip, Nat. Commun. 10, 4435 (2019).

[14] C. Errando-Herranz, E. Schöll, M. Laini, S. Gyger, A. W. Elshaari, A. Branny, U. Wennberg, S. Barbat, T. Renaud, M. Brotons-Gisbert, C. Bonato, B. D. Gerardot, V. Zwiller, and K. D. Jöns, On-chip single photon emission from a waveguide-coupled two-dimensional semiconductor, arXiv:2002.07657 (2020).

[15] N. Somaschi, V. Giesz, L. De Santis, J. C. Loredo, M. P. Almeida, G. Hornecker, S. L. Portalupi, T. Grange, C. Antón, J. Demory, C. Gómez, I. Sagnes, N. D. LanzillottiKimura, A. Lemaítre, A. Auffeves, A. G. White, L. Lanco, and P. Senellart, Near-optimal single-photon sources in the solid state, Nat. Photonics 10, 340 (2016).

[16] X. Ding, Y. He, Z.-C. Duan, N. Gregersen, M.-C. Chen, S. Unsleber, S. Maier, C. Schneider, M. Kamp, S. Höfling, C.-Y. Lu, and J.-W. Pan, On-Demand Single Photons with High Extraction Efficiency and Near-Unity 
Indistinguishability from a Resonantly Driven Quantum Dot in a Micropillar, Phys. Rev. Lett. 116, 020401 (2016).

[17] J. Liu, R. Su, Y. Wei, B. Yao, S. F. C. da Silva, Y. Yu, J. Iles-Smith, K. Srinivasan, A. Rastelli, J. Li, and X. Wang, A solid-state source of strongly entangled photon pairs with high brightness and indistinguishability, Nat. Nanotechnol. 14, 586 (2019).

[18] R. J. Coles, D. M. Price, J. E. Dixon, B. Royall, E. Clarke, P. Kok, M. S. Skolnick, A. M. Fox, and M. N. Makhonin, Chirality of nanophotonic waveguide with embedded quantum emitter for unidirectional spin transfer, Nat. Commun. 7, 11183 (2016).

[19] M. Davanco, J. Liu, L. Sapienza, C.-Z. Zhang, J. V. De Miranda Cardoso, V. Verma, R. Mirin, S. W. Nam, L. Liu, and K. Srinivasan, Heterogeneous integration for on-chip quantum photonic circuits with single quantum dot devices, Nat. Commun. 8, 889 (2017).

[20] J. Q. Grim, A. S. Bracker, M. Zalalutdinov, S. G. Carter, A. C. Kozen, M. Kim, C. S. Kim, J. T. Mlack, M. Yakes, B. Lee, and D. Gammon, Scalable in operando strain tuning in nanophotonic waveguides enabling three-quantum-dot superradiance, Nat. Mater. 18, 963 (2019).

[21] R. Uppu, H. T. Eriksen, H. Thyrrestrup, A. D. Uğurlu, Y. Wang, S. Scholz, A. D. Wieck, A. Ludwig, M. C. Löbl, R. J. Warburton, P. Lodahl, and L. Midolo, On-chip deterministic operation of quantum dots in dual-mode waveguides for a plug-and-play single-photon source, Nat. Commun. 11, 3782 (2020).

[22] S. Slussarenko and G. J. Pryde, Photonic quantum information processing: A concise review, Appl. Phys. Rev. 6, 041303 (2019).

[23] K. Takemoto, Y. Nambu, T. Miyazawa, Y. Sakuma, T. Yamamoto, S. Yorozu, and Y. Arakawa, Quantum key distribution over $120 \mathrm{~km}$ using ultrahigh purity single-photon source and superconducting single-photon detectors, Sci. Rep. 5, 14383 (2015).

[24] H.-K. Lo, M. Curty, and B. Qi, Measurement-DeviceIndependent Quantum Key Distribution, Phys. Rev. Lett. 108, 130503 (2012).

[25] J. C. Loredo, M. A. Broome, P. Hilaire, O. Gazzano, I. Sagnes, A. Lemaitre, M. P. Almeida, P. Senellart, and A. G. White, Boson Sampling with Single-Photon Fock States from a Bright Solid-State Source, Phys. Rev. Lett. 118, 130503 (2017).

[26] H. Wang, J. Qin, X. Ding, M.-C. Chen, S. Chen, X. You, Y.-M. He, X. Jiang, L. You, Z. Wang, C. Schneider, J. J. Renema, S. Höfling, C.-Y. Lu, and J.-W. Pan, Boson Sampling with 20 Input Photons and a 60-Mode Interferometer in a $10^{14}$-Dimensional Hilbert Space, Phys. Rev. Lett. 123, 250503 (2019).

[27] I. Schwartz, D. Cogan, E. R. Schmidgall, Y. Don, L. Gantz, O. Kenneth, N. H. Lindner, and D. Gershoni, Deterministic generation of a cluster state of entangled photons, Science 354, 434 (2016).

[28] D. Scerri, R. N. E. Malein, B. D. Gerardot, and E. M. Gauger, Frequency-encoded linear cluster states with coherent Raman photons, Phys. Rev. A 98, 022318 (2018).

[29] M. Gimeno-Segovia, T. Rudolph, and S. E. Economou, Deterministic Generation of Large-Scale Entangled Photonic
Cluster State from Interacting Solid State Emitters, Phys. Rev. Lett. 123, 070501 (2019).

[30] L.-M. Duan, M. D. Lukin, J. I. Cirac, and P. Zoller, Longdistance quantum communication with atomic ensembles and linear optics, Nature 414, 413 (2001).

[31] J. I. Cirac, A. K. Ekert, S. F. Huelga, and C. Macchiavello, Distributed quantum computation over noisy channels, Phys. Rev. A 59, 4249 (1999).

[32] H. J. Kimble, The quantum Internet, Nature 453, 1023 (2008).

[33] T. Hayashi, T. Taru, O. Shimakawa, T. Sasaki, and E. Sasaoka, Design and fabrication of ultra-low crosstalk and low-loss multi-core fiber, Opt. Express 19, 16576 (2011).

[34] J. Sakaguchi, Y. Awaji, N. Wada, A. Kanno, T. Kawanishi, T. Hayashi, T. Taru, T. Kobayashi, and M. Watanabe, in Optical Fiber Communication Conference/National Fiber Optic Engineers Conference 2011 (Optical Society of America, Los Angeles, California, United States, 2011), p. PDPB6.

[35] T. A. Birks, B. J. Mangan, A. Díez, J. L. Cruz, and D. F. Murphy, "Photonic lantern" spectral filters in multi-core fibre, Opt. Express 20, 13996 (2012).

[36] Y. Ding, D. Bacco, K. Dalgaard, X. Cai, X. Zhou, K. Rottwitt, and L. K. Oxenløwe, High-dimensional quantum key distribution based on multicore fiber using silicon photonic integrated circuits, npj Quantum Inf. 3, 25 (2017).

[37] G. Munoz-Matutano, D. Barrera, C. R. Fernandez-Pousa, R. Chulia-Jordan, J. Martinez-Pastor, I. Gasulla, L. Seravalli, G. Trevisi, P. Frigeri, and S. Sales, Parallel recording of single quantum dot optical emission using multicore fibers, IEEE Photonics Technol. Lett. 28, 1257 (2016).

[38] D. Dalacu, A. Kam, D. G. Austing, X. Wu, J. Lapointe, G. C. Aers, and P. J. Poole, Selective-area vapour-liquidsolid growth of InP nanowires, Nanotechnology 20, 395602 (2009).

[39] P. J. Poole, D. Dalacu, X. Wu, J. Lapointe, and K. Mnaymneh, Interplay between crystal phase purity and radial growth in InP nanowires, Nanotechnology 23, 385205 (2012).

[40] D. Dalacu, P. J. Poole, and R. L. Williams, Nanowire-based sources of non-classical light, Nanotechnology 30, 232001 (2019).

[41] D. Cogan, O. Kenneth, N. H. Lindner, G. Peniakov, C. Hopfmann, D. Dalacu, P. J. Poole, P. Hawrylak, and D. Gershoni, Depolarization of Electronic Spin Qubits Confined in Semiconductor Quantum Dots, Phys. Rev. X 8, 041050 (2018).

[42] M. A. M. Versteegh, M. E. Reimer, K. D. Jöns, D. Dalacu, P. J. Poole, A. Gulinatti, A. Giudice, and V. Zwiller, Observation of strongly entangled photon pairs from a nanowire quantum dot, Nat. Commun. 5, 5298 (2014).

[43] K. D. Jöns, L. Schweickert, M. A. M. Versteegh, D. Dalacu, P. J. Poole, A. Gulinatti, A. Giudice, V. Zwiller, and M. E. Reimer, Bright nanoscale source of deterministic entangled photon pairs violating Bell's inequality, Sci. Rep. 7, 1700 (2017).

[44] D. Dalacu, K. Mnaymneh, J. Lapointe, X. Wu, P. J. Poole, G. Bulgarini, V. Zwiller, and M. E. Reimer, Ultraclean emission from InAsP quantum dots in defect-free wurtzite InP nanowires, Nano Lett. 12, 5919 (2012). 
[45] M. E. Reimer, G. Bulgarini, N. Akopian, M. Hocevar, M. B. Bavinck, M. A. Verheijen, E. P. A. M. Bakkers, L. P. Kouwenhoven, and V. Zwiller, Bright single-photon sources in bottom-up tailored nanowires, Nat. Commun. 3, 737 (2012).

[46] G. Bulgarini, M. E. Reimer, M. Bouwes Bavinck, K. D. Jöns, D. Dalacu, P. J. Poole, E. P. A. M. Bakkers, and V. Zwiller, Nanowire waveguides launching single photons in a Gaussian mode for ideal fiber coupling, Nano Lett. 14, 4102 (2014).

[47] P. Laferrière, E. Yeung, L. Giner, S. Haffouz, J. Lapointe, G. C. Aers, P. J. Poole, R. L. Williams, and D. Dalacu, Multiplexed single-photon source based on multiple quantum dots embedded within a single nanowire, Nano Lett. 20, 3688 (2020).

[48] I. E. Zadeh, A. W. Elshaari, K. D. Jns, A. Fognini, D. Dalacu, P. J. Poole, M. E. Reimer, and V. Zwiller, Deterministic integration of single photon sources in silicon based photonic circuits, Nano Lett. 16, 2289 (2016).

[49] A. W. Elshaari, E. Büyüközer, I. E. Zadeh, T. Lettner, P. Zhao, E. Schöll, S. Gyger, M. E. Reimer, D. Dalacu, P. J. Poole, K. D. Jöns, and V. Zwiller, Strain-tunable quantum integrated photonics, Nano Lett. 18, 7969 (2018).

[50] G. Ballesteros, R. Proux, C. Bonato, and B. Gerardot, readPTU: A PYTHON library to analyse time tagged time resolved data, J. Instrum. 14, T06011 (2019).

[51] T. Flissikowski, A. Betke, I. A. Akimov, and F. Henneberger, Two-Photon Coherent Control of a Single Quantum Dot, Phys. Rev. Lett. 92, 227401 (2004).

[52] S. Boyle, A. Ramsay, A. Fox, and M. Skolnick, Twocolor two-photon Rabi oscillation of biexciton in single InAs/GaAs quantum dot, Phys. E: Low-Dimensional Syst. Nanostruct. 42, 2485 (2010).

[53] H. Jayakumar, A. Predojević, T. Huber, T. Kauten, G. S. Solomon, and G. Weihs, Deterministic Photon Pairs and Coherent Optical Control of a Single Quantum Dot, Phys. Rev. Lett. 110, 135505 (2013).

[54] M. E. Reimer, G. Bulgarini, A. Fognini, R. W. Heeres, B. J. Witek, M. A. M. Versteegh, A. Rubino, T. Braun, M. Kamp, S. Höfling, D. Dalacu, J. Lapointe, P. J. Poole, and V. Zwiller, Overcoming power broadening of the quantum dot emission in a pure wurtzite nanowire, Phys. Rev. B 93, 195316 (2016).

[55] P. E. Kremer, A. C. Dada, P. Kumar, Y. Ma, S. Kumar, E. Clarke, and B. D. Gerardot, Strain-tunable quantum dot embedded in a nanowire antenna, Phys. Rev. B 90, 201408(R) (2014).

[56] Y. Chen, I. E. Zadeh, K. D. Jns, A. Fognini, M. E. Reimer, J. Zhang, D. Dalacu, P. J. Poole, F. Ding, V. Zwiller, and O. G. Schmidt, Controlling the exciton energy of a nanowire quantum dot by strain fields, Appl. Phys. Lett. 108, 182103 (2016).

[57] A. Fiset-Cyr, D. Dalacu, S. Haffouz, P. J. Poole, J. Lapointe, G. C. Aers, and R. L. William, In-situ tuning of individual position-controlled nanowire quantum dots via laserinduced intermixing, Appl. Phys. Lett. 113, 053105 (2018).

[58] A. V. Kuhlmann, J. Houel, A. Ludwig, L. Greuter, D. Reuter, A. D. Wieck, M. Poggio, and R. J. Warburton, Charge noise and spin noise in a semiconductor quantum device, Nat. Phys. 9, 570 (2013).
[59] A. V. Kuhlmann, J. H. Prechtel, J. Houel, A. Ludwig, D. Reuter, A. D. Wieck, and R. J. Warburton, Transformlimited single photons from a single quantum dot, Nat. Commun. 6, 8204 (2015).

[60] R. B. Patel, A. J. Bennett, I. Farrer, C. A. Nicoll, D. A. Ritchie, and A. J. Shields, Two-photon interference of the emission from electrically tunable remote quantum dots, Nat. Photonics 4, 632 (2010).

[61] M. Reindl, K. D. Jns, D. Huber, C. Schimpf, Y. Huo, V. Zwiller, A. Rastelli, and R. Trotta, Phonon-assisted twophoton interference from remote quantum emitters, Nano Lett. 17, 4090 (2017).

[62] B. Kambs and C. Becher, Limitations on the indistinguishability of photons from remote solid state sources, New J. Phys. 20, 115003 (2018).

[63] D. Huber, M. Reindl, S. F. Covre da Silva, C. Schimpf, J. Martín-Sánchez, H. Huang, G. Piredda, J. Edlinger, A. Rastelli, and R. Trotta, Strain-Tunable GaAs Quantum Dot: A Nearly Dephasing-Free Source of Entangled Photon Pairs on Demand, Phys. Rev. Lett. 121, 033902 (2018).

[64] A. V. Kuhlmann, J. Houel, D. Brunner, A. Ludwig, D. Reuter, A. D. Wieck, and R. J. Warburton, A dark-field microscope for background-free detection of resonance fluorescence from single semiconductor quantum dots operating in a set-and-forget mode, Rev. Sci. Instrum. 84, 073905 (2013).

[65] G. Jundt, L. Robledo, A. Högele, S. Fält, and A. Imamoğlu, Observation of Dressed Excitonic States in a Single Quantum Dot, Phys. Rev. Lett. 100, 177401 (2008).

[66] B. D. Gerardot, D. Brunner, P. A. Dalgarno, K. Karrai, A. Badolato, P. M. Petroff, and R. J. Warburton, Dressed excitonic states and quantum interference in a three-level quantum dot ladder system, New J. Phys. 11, 013028 (2009).

[67] P.-L. Ardelt, M. Koller, T. Simmet, L. Hanschke, A. Bechtold, A. Regler, J. Wierzbowski, H. Riedl, J. J. Finley, and K. Müller, Optical control of nonlinearly dressed states in an individual quantum dot, Phys. Rev. B 93, 165305 (2016)

[68] F. Hargart, K. Roy-Choudhury, T. John, S. L. Portalupi, C. Schneider, S. Höfling, M. Kamp, S. Hughes, and P. Michler, Probing different regimes of strong field light-matter interaction with semiconductor quantum dots and few cavity photons, New J. Phys. 18, 123031 (2016).

[69] S. Bounouar, M. Strauß, A. Carmele, P. Schnauber, A. Thoma, M. Gschrey, J.-H. Schulze, A. Strittmatter, S. Rodt, A. Knorr, and S. Reitzenstein, Path-Controlled Time Reordering of Paired Photons in a Dressed Three-Level Cascade, Phys. Rev. Lett. 118, 233601 (2017).

[70] M. O. Scully and M. S. Zubairy, Quantum Optics (Cambridge University Press, Cambridge, 1997).

[71] M. Glässl, A. M. Barth, K. Gawarecki, P. Machnikowski, M. D. Croitoru, S. Lüker, D. E. Reiter, T. Kuhn, and V. M. Axt, Biexciton state preparation in a quantum dot via adiabatic rapid passage: Comparison between two control protocols and impact of phonon-induced dephasing, Phys. Rev. B 87, 085303 (2013).

[72] T. Kaldewey, S. Lüker, A. V. Kuhlmann, S. R. Valentin, J.-M. Chauveau, A. Ludwig, A. D. Wieck, D. E. Reiter, 
T. Kuhn, and R. J. Warburton, Demonstrating the decoupling regime of the electron-phonon interaction in a quantum dot using chirped optical excitation, Phys. Rev. B 95, 241306(R) (2017).

[73] P.-L. Ardelt, L. Hanschke, K. A. Fischer, K. Müller, A. Kleinkauf, M. Koller, A. Bechtold, T. Simmet, J. Wierzbowski, H. Riedl, G. Abstreiter, and J. J. Finley,
Dissipative preparation of the exciton and biexciton in selfassembled quantum dots on picosecond time scales, Phys. Rev. B 90, 241404(R) (2014).

[74] S. Bounouar, M. Müller, A. M. Barth, M. Glässl, V. M. Axt, and P. Michler, Phonon-assisted robust and deterministic two-photon biexciton preparation in a quantum dot, Phys. Rev. B 91, 161302(R) (2015). 